\title{
Complex Power System for Lithium Batteries and Lead-Acid Batteries
}

\author{
Hendrick , Chen-Chai Tsai, Jyun-Teng Jheng, Zhi-Hao Wang and Gwo-Jia Jong \\ National Kaohsiung University of Applied Sciences, No.415, Jiangong Rd., Sanmin Dist., Kaohsiung City 807, Taiwan
}

\begin{abstract}
This paper discusses about the power system, and focus on complex power system applications on the public transportation. The objective of this research is to improve power system to achieve green energy applications. Energy depletion is a global problem for people who live on the Earth and we have to face this problem. So before energy depletion occurs, we should develop more energy alternatives. In this paper, we go through two experimental verification of complex power systems. The first part is to establish a platform for static electricity complex experiments. In the second part, using vehicle test platform for dynamic test. Finally, by creating a motor drive mode simulate actual conditions to enhance the accuracy of the experimental results. According to the results we can know the power system after being modified can improve overall system performance.
\end{abstract}

\section{Introduction}

Currently, many researchers improve all kinds of energy sources. A lot of energy was limited by natural condition in long time period. It makes some countries difficult to develop efficiently. Some energy are still unable to break through science and technology by using renewable energy but they still get some weakness that need to solve, for example the conversion efficiency of solar panels is too low, the plate is also limited, wind power due to lack of stable wind band, hydroelectric because the terrain cause cannot store enough water to generate electricity and nuclear energy because radioactive material also cannot become applicable energy alternatives. In the case of these resources are unable to successfully use, complex power system is a very important role. It has many advantages, such as there is no the terrain restrictions, accepts multiple power sources, high conversion efficiency, without noise, etc. So complex power system in recent years become the focus of energy alternatives.

\section{Research method}

In this paper, the experiment method was divided into two parts, which are static test and dynamic test. The following will explain the methods.

\subsection{Static test}

In the first part of this experiment is focused to test the design of power system. It is really need to do for getting the real condition when this system is used. in the power system it have some elements such as power converter that used to generate the energy resource from power resource 1 and power resource $\mathrm{N}$, sensor and a microcontroller as the main element, algorithms to control the energy system then a power supply and load. Beside that giving a light signal is the most impotent thing as the status to indicate complete test already finished. Completely, it will fig out as the following bellow in Fig. 1.

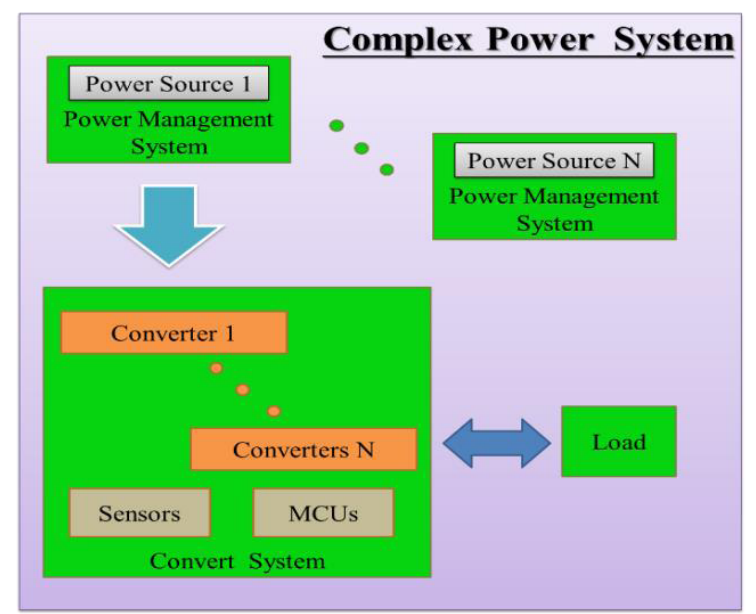

Figure 1. System block diagram 


\subsection{Dynamic test}

The second part of the experiment, we want to know further result when the system that we already create to use for the real application. We use vehicle as testing platform to test complex power system, and with reference to the urban driving mode, simulate the motor load when vehicle driving on the road. We use the experiment by using many kind variations and modes including acceleration driving, deceleration driving, constant speed and slide driving. Apparently, all of these type use different power source consumption. From fig. 2 it shows that the red line is the power consumption and the blue one as the speed of vehicle. When the vehicle speed increase smoothly so the power consumption will rise dramatically. Whereas when the speed growth rapidly so the power usage only smoothly increase then when the vehicle have constant speed so power consumption will growth slowly. On the other hands, the consumption of power will be constant when the speed of vehicle is decline. According to this map, consumption of power is depend on speed of vehicle. As power source, it used the Lithium Batteries and Lead-Acid Batteries. So the system has two inputs and one output.

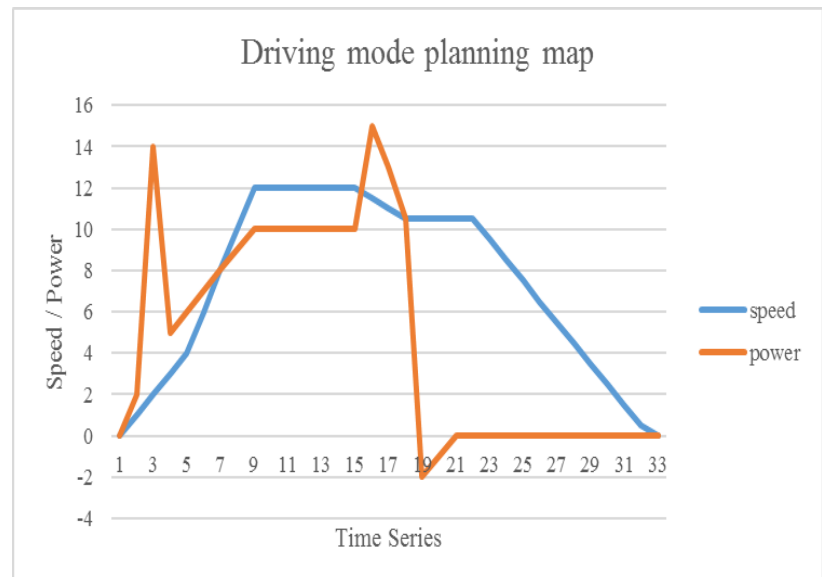

Figure 2. Driving mode planning map.

\section{System architecture}

The system architecture is divided into part of hardware and algorithms. The following will explain it.

\subsection{Lithium-ion batteries}

Lithium ion batteries are all secondary batteries among the highest energy density, power density, and also the longest life expectancy. The Battery based on lithium-ion battery cathode materials can be divided into different lithium cobalt batteries, lithium nickel batteries, lithiumnickel-cobalt-manganese and lithium iron phosphate battery. Lithium battery has a stable output voltage curve, so it is more convenient to use the back-end design.

Basically, lithium-ion battery have any characteristic that appropriate as energy resource in our experiment, in Fig. 3 shows the curve of this battery. We can see that the red line is the voltage curve, most of the voltage at $3.7 \mathrm{~V}$. When the battery is fully charge the voltage will increase from 3.7 to maximum voltage $4.2 \mathrm{~V}$. Which can note characteristic point, which is the transition point with a constant current constant voltage, which it helps algorithm design.

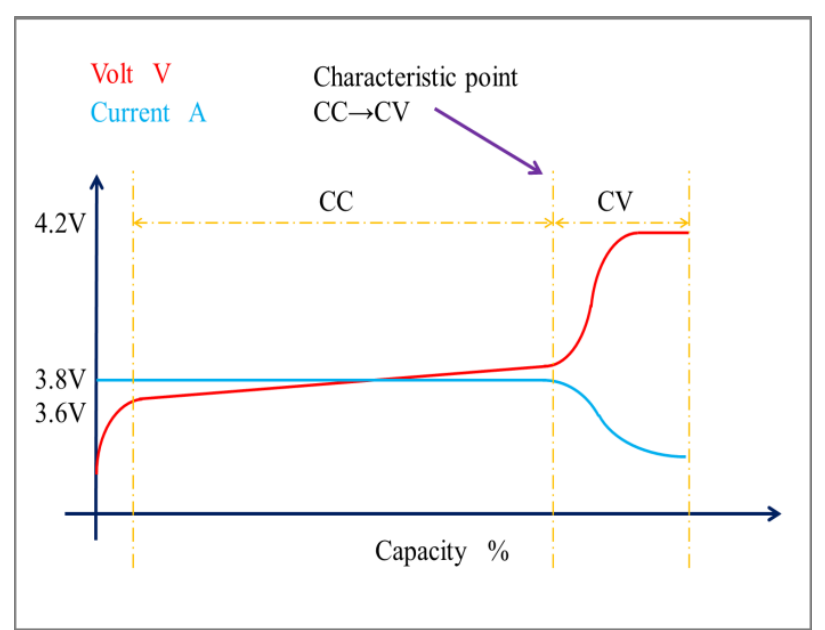

Figure 3. Characteristic curve.

\subsection{Lead-acid batteries}

In this experiment the use of lead-acid battery voltage is $12 \mathrm{~V}$ which has $22 \mathrm{Ah}$ nominal capacity. The battery is a series consisting of six $2 \mathrm{~V}$ single cells. In the part of the power output capacity of the battery maximum continuous output current is $330 \mathrm{~A}$, and also indicate the maximum continuous output power is $3.96 \mathrm{~kW}$. [1]

\subsection{Power converter}

Power conversion module is mainly composed of halfbrick DC converter. Since each battery in different output power will correspond to different output voltages to adjust the final output voltage through the complex power system. The complex power system can arbitrarily set the output voltage and the maximum output current for different needs. At the input of the module, with the use of diode allows converters to be protected.

DC converter used in this experiment is shown in Fig 4 , the input and output voltage ranges are $9 \sim 60 \mathrm{~V}$ and 0 $\sim 60 \mathrm{~V}$, maximum input and output current is $40 \mathrm{~A}$, the maximum module efficiency of up to $97 \%$ and an average efficiency of $95 \%$ outside. Its output current can be used with an external circuit design to achieve constant current output. The module is also the composite power system module inside the core element, its size and weight to be able to withstand the power of $1.2 \mathrm{~kW}$ [2].

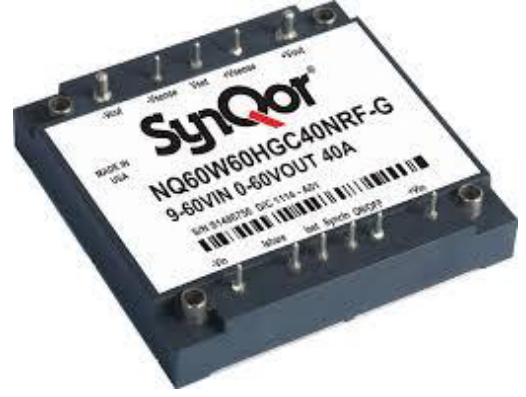

Figure 4. DC Converter 


\subsection{Microcontrollers}

A microcontroller usedin experiment is manufactured by Microchip controlled controller (PIC18F series), which have built in the $\mathrm{AD}$ converter, serial communication module and low power consumption, etc.

We use microcontroller as the main control in this system. Microcontroller will determine base on algorithm that we already create. we wrote the algorithm into the controller, which can be set freely to control on different input power based on the user's parameters. Using 12-bit $\mathrm{AD}$ converter to precisely measure the amount of electrical signals within the system, provide feedback to the algorithm.

\subsection{Sensors}

This experiment used two kinds of sensor such as a voltage sensor and a current sensor. Voltage sensor measurement is made by resistance dividing the signal. The resistance value must be a very high value in order to maintain low power consumption and accuracy. Current sensor using a hall sensor, because the amount of non- contact measurement of the loss can be avoided, but must provide a negative supply.

The following equation based experiments converted voltage and current signals.

Where $r$ is AD conversion result.

$$
\begin{aligned}
& V(R)=0.0513 * \mathrm{R}+3.3204 \\
& I(R)=0.0235 * R-1.7977
\end{aligned}
$$

\subsection{Algorithms}

In this experiment, the key point is that the algorithms constant voltage and constant current converter. In constant current mode, voltage can be changed. In constant voltage mode, current can be changed. So we have to make lithium-ion batteries operated in two modes to control the output of lead-acid batteries.

Because the characteristics of lithium batteries are not suitable for suddenly output but suitable for stable output, so we will limit its maximum power point, and lead-acid batteries for load changes sharply. We use the complex power system to combined two different characteristics power source.

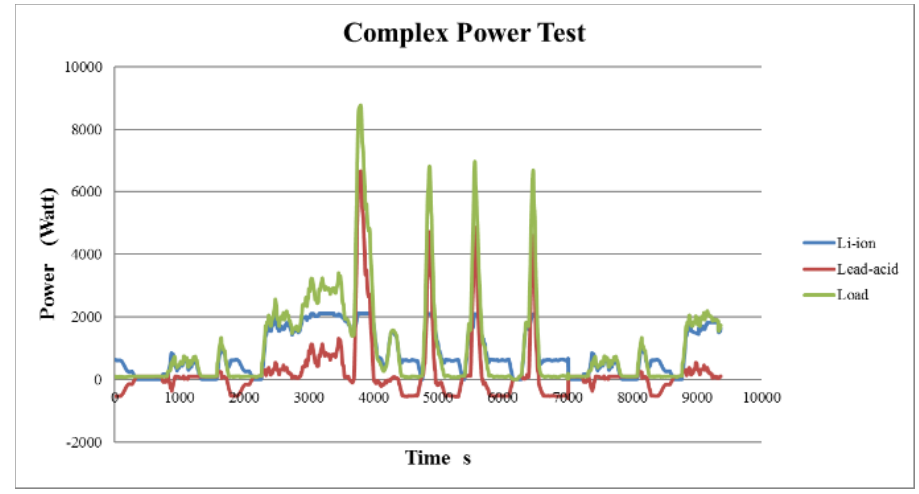

Figure 5. Characteristic curve.

Our system test results are shown in Fig. 5, the blue line represents a lithium-ion battery power. Red is the lead-acid battery power and green is the load power (total power).

\section{Conclusion}

In the experiment, we established a prototype for complex power system and found a way to control the system. Algorithm limits the maximum output power of lithium batteries, first to protect the lithium battery to maintain the optimum operating point, the second can make overall system to operate in a higher efficiency point and the third is to reach a complex power system performance.

In fact, we can add a variety of other sources of electricity, but after comparison, we chose the lead-acid batteries and lithium batteries as a power source. Both of power sourcenot only can quickly provide a large output power but also has a low cost. The Complex power system can quickly build. The complex power system will be more suitable for public transportation.

\section{References}

1. REC22-12, 2015.

http://www.yuasa.com.tw/_english/03_vrla/03_detail .php?vd=51.

2. NQ60W60HGC40NRF-G:Half-Brick, http://parts.arrow.com/item/detail/synqor/nq60w60h gc40nrf-g\#GGzn

3. Au-Yong-Tien-Tuck, "The Research and Development of Vechicle's Fuel Cell System Control Module with Performances Improved Mechanism", National Kaohsiung University of Applied Sciences, Jul. 2014, Page(s): 61-63.

4. Hong Wen HE, YingQi Zhang, Fan Wan, 2008, "Control Strategies Design for a Fuel Cell Hybrid Electric Vehicle," IEEE Vehicle Power and Propulsion Conference (VPPC).

5. V. H. Johnson, "Battery Performance Models in ADVISOR," Journal of Power Sources, 110, pp. 321-329, Aug. 2002.

6. D. P. Xu, L. F. Wang, and J. A. Yang, "Research on Li-ion Battery Management System," Proceedings of 
2010 International Conference on Electrical and Control Engineering, pp. 4106-4109, Jun. 2010.

7. Y. C. Hsieh and C. S. Huang, "Li-ion Battery Charger Based on Digitally Controlled Phase-shifted Full-bridge Converter," IET Power Electron, 4(2), pp. 242-247, Feb. 2011. 